Document downloaded from:

http://hdl.handle.net/10251/64466

This paper must be cited as:

San Blas Oltra, AA.; Boria Esbert, VE.; Gimeno Martinez, B.; Cogollos Borras, S. (2015). Design of compensated multiport waveguide junctions considering mechanization effects. AEU - International Journal of Electronics and Communications. 69(1):328-331. doi:10.1016/j.aeue.2014.10.003.



The final publication is available at

http://dx.doi.org/10.1016/j.aeue.2014.10.003

Copyright Elsevier

Additional Information 


\title{
Design of Compensated Multiport Waveguide Junctions considering Mechanization Effects
}

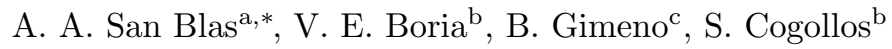 \\ ${ }^{a}$ Departamento de Ingeniería de Comunicaciones, Universidad Miguel Hernández de Elche, \\ Avenida de la Universidad s/n, E-03203 Elche (Spain) \\ ${ }^{b}$ Departamento de Comunicaciones, Universidad Politécnica de Valencia, Valencia (Spain) \\ ${ }^{c}$ Departamento de Física Aplicada y Electromagnetismo, Universidad de Valencia, Valencia \\ (Spain)
}

\begin{abstract}
A new tool for the rigorous and efficient design of compensated multiport waveguide junctions, considering the mechanization effects due to low-cost manufacture techniques, is presented. Several new designs for a great variety of key components, present in modern microwave and millimetre-wave equipment, are proposed taking into account the introduction of rounded corners in the rectangular waveguide access ports. The new implemented tool permits to control and compensate for the potential degradation of the wide-band performance of such components prior to their fabrication, thus achieving an optimal design. All the presented results have been successfully validated by comparing the obtained simulated data with the results provided by a commercial software based on the finite-element method.
\end{abstract}

Keywords: Multiport waveguide junctions, mechanization effects, full-wave methods.

\section{Introduction}

Wide-band multiport rectangular waveguide junctions are a topic of high interest in the field of modern microwave and millimetre-wave equipment, and they have been intensively investigated in the recent years [1, 2], [3]. These

\footnotetext{
* Corresponding author

Email address: aasanblas@umh.es (A. A. San Blas)
}

Published in International Journal of Electronics and Communications vol. 69, pp. 328-331, 2015. 
components are widely used in many telecommunication devices, such as beam forming networks, power dividers or manifold multiplexers, when multiple channels, low insertion losses, and a broadband operation performance are required. Traditionally, the usable bandwidth of these multiport junctions has been optimized by compensating the structure using metallic insets, such as square prisms, pyramid tuning stubs, and cylindrical posts [4, [5], 6], 7].

Moreover, it is usual to resort to low-cost waveguide device fabrication techniques, such as computer-controlled milling, electroforming or die-casting, in order to manufacture these optimized components. A common drawback of these previous techniques is the introduction of rounded corners in the crosssection of the waveguide access ports of the cited junctions, thus deteriorating the expected electrical response of the designed device and, therefore, limiting its desired wide-band performance [2]. Nevertheless, if computer-aided design (CAD) tools could accurately consider these negative manufacture effects in the analysis stage, it would be possible to compensate them and achieve optimum electrical designs of such components easily implementable via low-cost fabrication techniques. As a consequence, there is an ever-increasing interest in compensated multiport junctions CAD tools enabling the accurate design of these components, prior to their final hardware implementation using low-cost manufacture procedures.

In this letter, a novel full-wave CAD tool is proposed for the accurate and efficient design of compensated multiport waveguide junctions, taking into account the mechanization effects introduced by the low-cost production methods aforementioned. On the one hand, the proposed CAD tool permits the microwave designer to predict the electrical performance of the final manufactured device as a function of the radius of the considered rounded corners. On the other hand, it also enables to compensate for the degradation introduced in the frequency response of the device by redesigning the junction (i.e. modifying the position and/or the dimensions of the compensating stub) in order to achieve an optimum broadband operation.

In order to fully validate the presented CAD tool, new designs for a great 
variety of key components present in many communication satellite subsystems are provided. In these devices, the effect of the rounded corners is considered, and partial-height cylindrical metallic posts are used as compensating elements. The first examples consist of new designs concerning compensated $H$-plane Tjunctions (see Fig. 1) and right-angled bends. A more complex five-port device, specifically a compensated turnstile junction, considering these mechanization effects is also reported.

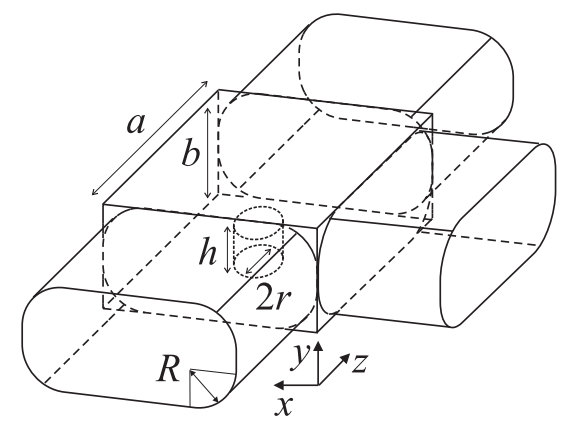

Figure 1: $H$-plane T-junction compensated with a partial-height cylindrical metallic post of radius $r$ and height $h$. Rounded corners of radius $R$ have been introduced in the waveguide access ports to take into account the mechanization effects due to low-cost production.

It is important to emphasize that this work provides, for the first time to the authors' knowledge, a rigorous combination of 2-D and 3-D Boundary IntegralResonant Mode Expansion (BI-RME) techniques [2, 8 for the efficient analysis and design of compensated multiport waveguide junctions considering mechanization effects. To this respect, note that the novelty of this work lies on the fact that previous works of the authors did not considered the presence of rounded corners in the analyzed multiport junctions (i.e. [3], 6], 9]) or did not deal with the design of compensated multiport junctions using the 3-D BIRME technique (i.e. [2]). As a consequence, the work developed in this letter, not only constitutes a significative extension of previous contributions, but also provides a novel and very efficient $\mathrm{CAD}$ tool aiming at improving the relative bandwidth of a great variety of compensated waveguide junctions considering mechanization effects. 


\section{Full-wave analysis of multiport waveguide junctions considering mechanization effects}

The implemented full-wave CAD tool is mainly based on the Boundary Integral-Resonant Mode Expansion (BI-RME) method. Concretely, the 2-D BIRME method [2] is used to characterize a rectangular waveguide with rounded corners. This multimodal technique has been combined with the 3-D BI-RME method [8], which yields a generalized admittance matrix of arbitrarily shaped 3 -D cavities, to finally provide a wide-band electromagnetic characterization of the whole structure.

To illustrate this concept in more detail, we start by considering the compensated $H$-plane T-junction depicted in Fig. 1. In order to analyze this component, the 3-D BI-RME method can be first employed to derive the generalized admittance matrix (GAM) of a rectangular cavity loaded with a partial-height cylindrical post, and considering standard rectangular waveguide access ports of dimensions $a \times b$. The elements of the GAM can be obtained as:

$$
\mathbf{Y}=\frac{1}{j k \eta} \mathbf{Y}^{\mathrm{A}}+\frac{j k}{\eta} \mathbf{Y}^{\mathrm{B}}+\frac{j k^{3}}{\eta} \sum_{i=1}^{M} \frac{\mathbf{y}^{(i)} \mathbf{y}_{\mathrm{T}}^{(i)}}{k_{i}^{2}-k^{2}}
$$

where $k$ is the wavenumber; $\eta$ is the wave impedance; matrices $\mathbf{Y}^{\mathrm{A}}$ and $\mathbf{Y}^{\mathrm{B}}$ are real, symmetric and frequency-independent; $k_{i}(i=1, \ldots, M)$ represent the first $M$ resonant wavenumbers of the shortcircuited cavity, and vectors $\mathbf{y}^{(i)}$ are related to the eigenvectors associated to $k_{i}$ (see [8] for more details).

Afterwards, the 2-D BI-RME method is applied to obtain both the modal chart and the expressions of the magnetic vector modal functions of a rectangular waveguide with rounded corners of radius $R$. To this aim, the rigorous technique detailed in [2] has been followed.

Next, the efficient integral equation technique described in [10] has been implemented to characterize a planar junction between a standard rectangular waveguide and a rectangular waveguide with rounded corners, in terms of a generalized impedance matrix.

Finally, all the obtained matrices (generalized impedance and admittance 


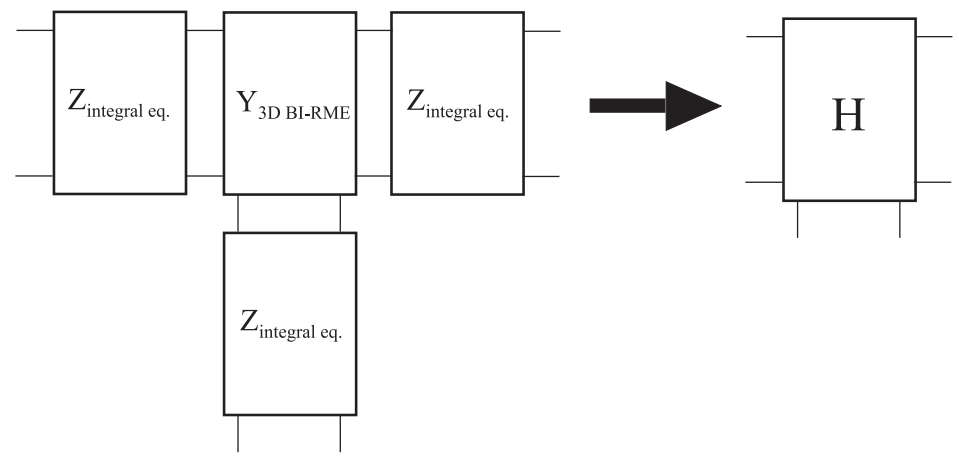

Figure 2: Connection of the generalyzed admittance and impedance matrices associated to the full-wave analysis of the $H$-plane T-junction shown in Fig. 1 The generalized impedance matrices $\mathbf{Z}$ are provided by the integral equation technique.

matrices) are properly connected, as shown in Fig. 2, to provide the electrical performance of the complete device. To this aim, it is worth mentioning that, for the sake of improving even more the computational efficiency of the implemented tool, an equivalent hybrid three-port immittance matrix ( $\mathbf{H}$ in Fig. 2 ) is derived for the characterization of the whole structure enabling only one matrix inversion per frequency point, and thus significantly reducing the overall CPU effort. Although this procedure has been detailed for the case of a three-port structure ( $H$-plane T-junction), it can be easily adapted to cope with two-port and fiveport structures, such as bends and turnstile junctions, respectively.

\section{Design of multiport waveguide junctions considering mechaniza- tion effects}

In this section, we present new designs concerning compensated $H$-plane right-angled bends and $H$-plane T-junctions, taking into account the mechanization effects introduced by the low-cost production of such components. The design of a more complex five-port device of a great technological interest, i.e. a compensated turnstile junction, is also discussed. In all cases, the obtained simulated results have been succesfully validated using the numerical data provided by a commercial finite-element method software. 
With the aim of achieving convergent results for all the new designs presented in this work, we have required to use 25 accessible modes in each waveguide port and 275 resonant modes in the rectangular cavity. In addition, the authors have verified that convergence properties of the method are not dependent on the post and rectangular cavity dimensions. Furthermore, for design purposes, the authors have followed the practical design procedures detailed in [11] to achieve an optimal electrical performance.

\subsection{Design of compensated $H$-plane right-angled bends}

A compensated $H$-plane right-angled bend is a two-port device that can be readily obtained starting from the three-port component depicted in Fig. 1 . after short-circuiting the port placed at $z=a$. Next, Fig. 3 shows the variation of the electrical performance of a new designed compensated $H$-plane rightangled bend considering the presence of rounded corners of radius $R$ in the two waveguide access ports. It is important to note that the curve $R=0$ stands for the case in which standard rectangular waveguides are considered and, therefore, the mechanization effect is not present. Besides, it is worth mentioning that the electrical performance of this new design with $R=0$ has been drastically improved with respect to the frequency response originally presented in $[3]$.

In this case, the bend is implemented in WR-90 standard waveguide ( $a=$ $22.86 \mathrm{~mm}, b=10.16 \mathrm{~mm}$ ), the radius and height of the compensating post are $r=0.5 \mathrm{~mm}$ and $h=8.75 \mathrm{~mm}$, respectively, and its relative position within the rectangular cavity (according to the axis system depicted in Fig. 1) is given by $x_{0}=z_{0}=15.5 \mathrm{~mm}$. Moreover, a very good agreement is observed between the obtained results and the numerical data provided by a finite-element method (FEM) commercial software.

It is possible to observe in Fig. 3 that the return losses of the bend deteriorates as the radius of the considered rounded corners increases. Lower values of the radius (e.g. $R=1$ or $R=2 \mathrm{~mm}$ ) do not change significantly the frequency response of the device. However, when higher values of the radius are considered (e.g. $R=4$ or $R=5 \mathrm{~mm}$ ), the usable bandwidth of the device and its 




Figure 3: Return losses of a compensated $H$-plane right-angled bend. Rounded corners of radius $R$ have been considered in the waveguide access ports.

overall electrical performance are seriously affected. In these cases, it is possible to compensate for this degradation by re-designing the compensating post using the proposed CAD tool. As it is represented in Fig. 4 using solid curves, an optimized electrical performance of the final manufactured device has been provided.

The new dimensions and relative positions of the post have been inserted in the figure. Finally, it is important to mention that the simulated results presented in this section were computed in just 0.02 seconds per frequency point (Intel Core i3@3.1 GHz - 4 GB RAM), thus demonstrating the high computational efficiency of the developed tool.

\subsection{Design of compensated H-plane T-junctions}

A new design concerning compensated $H$-plane T-junctions (see Fig. 1), considering the introduction of rounded corners of radius $R$ in all the rectangular waveguide access ports, is discussed in this section. The electrical performance of this component, which is widely used in modern diplexers and multiplexers, 


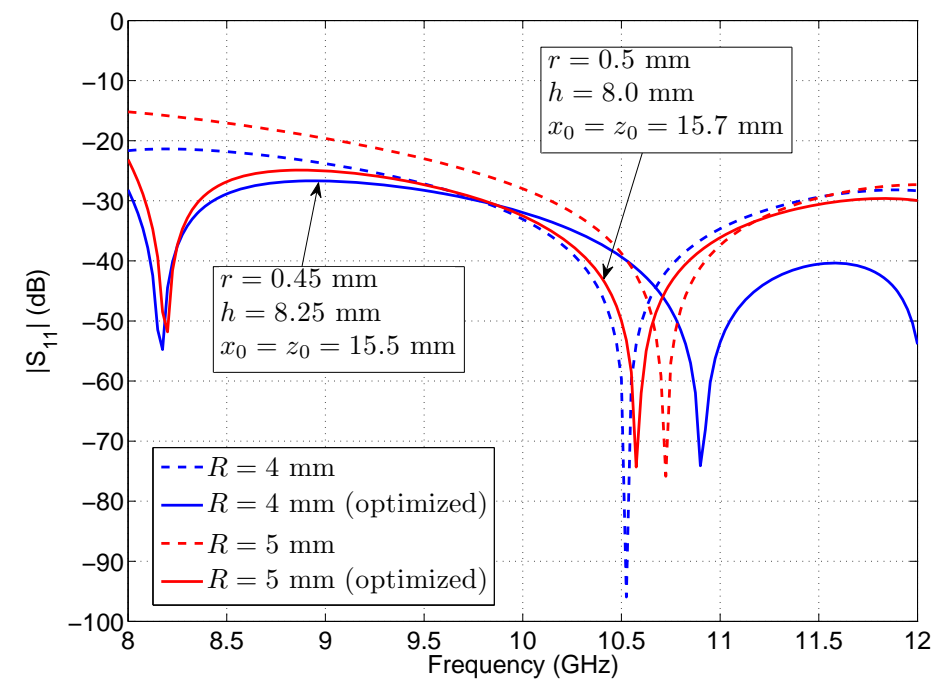

Figure 4: Return losses of a compensated $H$-plane right-angled bend. The compensating post has been re-designed to compensate for the degradation introduced by the rounded corners.

is investigated in Fig. 5 .

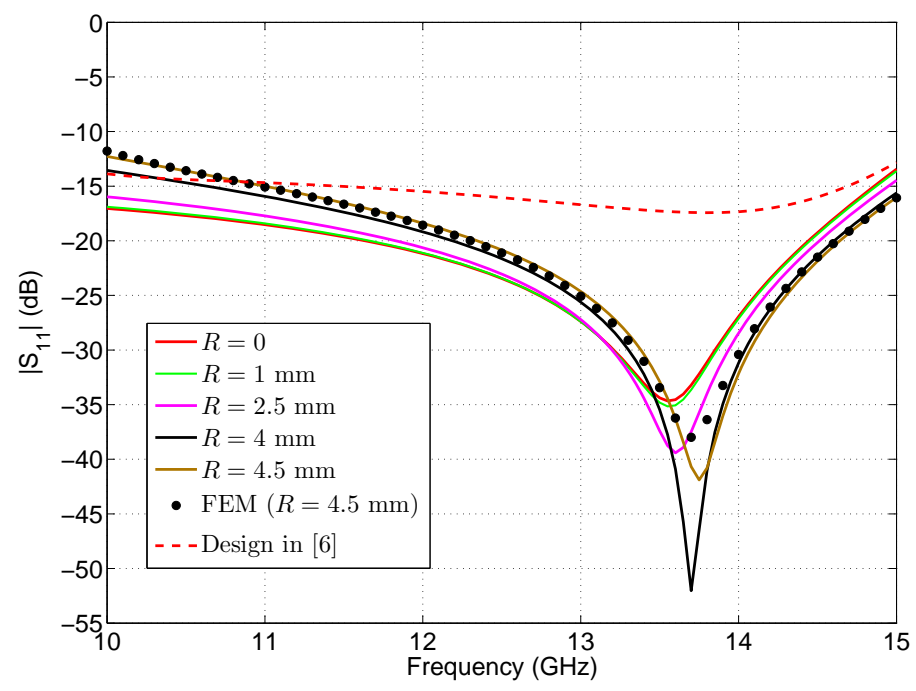

Figure 5: Electrical performance of a compensated $H$-plane T-junction (see Fig. 1 in terms of the radius $R$ of the considered rounded corners. 
The T-junction has been implemented in standard WR-75 waveguide ( $a=$ $19.05 \mathrm{~mm}, b=a / 2$ ), the radius of the compensating post is $r=0.454 \mathrm{~mm}$, its height is $h=8.8 \mathrm{~mm}$, and its relative position is $x_{0}=a / 2, z_{0}=11.525 \mathrm{~mm}$. It is important to note that, with respect to the previous results presented in [6], this new design (case for $R=0$ ) has significantly improved the return losses of the component.

With this design we conclude that, neither the wideband operation of the T-junction nor its overall electrical performance, are significantly affected by the introduction of the rounded corners and, therefore, further optimizations of the component are not required in this case. In fact, note that, even for higher values of the radius (e.g. $R=4.5 \mathrm{~mm}$ ), the usable bandwidth of the device under $-20 \mathrm{~dB}$ remains almost invariable (observing only a frequency shift). Finally, regarding the computational efficiency of the implemented CAD tool, the complete analysis of the T-junction only took 0.03 seconds per frequency point.

\subsection{Design of compensated turnstile junctions}

A turnstile junction is a five-port microwave network composed of four rectangular waveguide ports and one circular waveguide port (see the inset of Fig. 6). This component, which is a key element in modern orthomode transducers, can be obtained starting from the structure shown in Fig. 1, by adding a fourth rectangular port at $x=a$, and an input circular waveguide port at $y=b[9$.

The electrical performance of a compensated turnstile junction (circular waveguide of radius $r=1.26 \mathrm{~mm}$ ) implemented in WR-10 rectangular waveguide ( $a=2.54 \mathrm{~mm}, b=a / 2$ ), and considering the rounded corners effect in all the rectangular waveguide ports, is shown in Fig. 6 In contrast to the previous analyzed components, where a single cylindrical post was used as a compensating stub, this device is compensated using five piled-up cylindrical posts. The radii in $\mathrm{mm}$ of the piled-up cylindrical posts used in the design are (from bottom to top): $r_{1}=0.84, r_{2}=0.4, r_{3}=0.23, r_{4}=0.18, r_{5}=0.15$. The heights in $\mathrm{mm}$ of the posts are: $h_{1}=0.29, h_{2}=0.31, h_{3}=0.2, h_{4}=0.3, h_{5}=1.75$. 


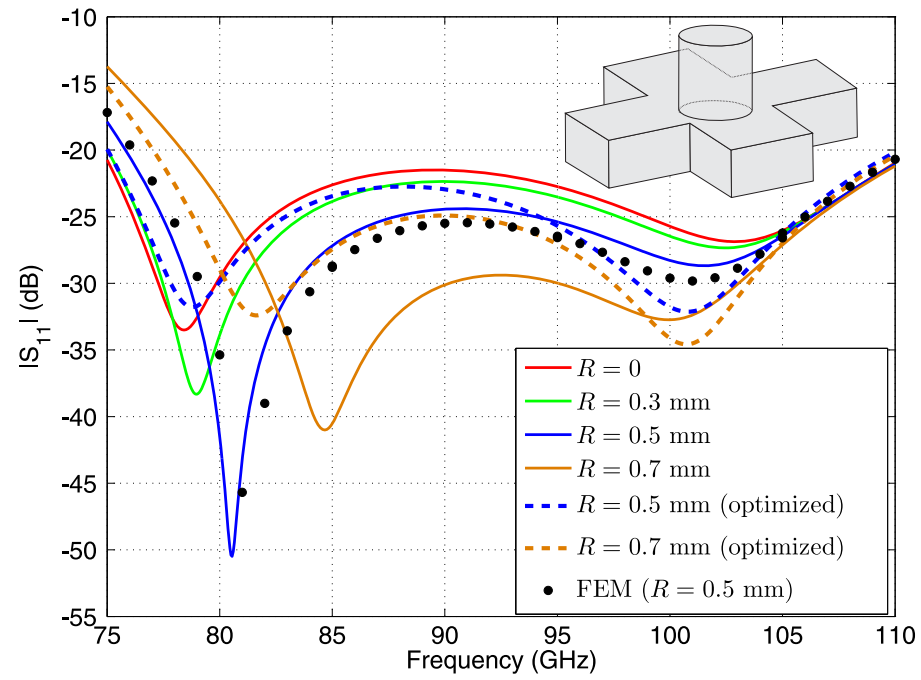

Figure 6: Return losses of a compensated turnstile junction as a function of the radius $R$ of the introduced rounded corners.

As in the previous discussed components, a degradation of the electrical response is observed as the radius of the rounded corners increases. In particular, note that the usable bandwidth below $-20 \mathrm{~dB}$ decreases for $R=0.5$ and $R=$ $0.7 \mathrm{~mm}$. Nevertheless, it is possible to compensate for this negative effect by redesigning the height of the top piled-up cylindrical post used to compensate the junction, as it is shown in the same Fig. 6 (dashed curves). Thanks to this new optimization, the usable bandwidth below $-20 \mathrm{~dB}$ for the case $R=0.5 \mathrm{~mm}$ is equal to the one achieved for the case $R=0$, while maintaining a good average return losses. Regarding the optimized case for $R=0.7 \mathrm{~mm}$, the relative bandwidth below $-20 \mathrm{~dB}$ has been increased in a $1.5 \%$ with respect to the original design. The new height of the top piled-up post used in these two new designs is $h_{5}=2.03 \mathrm{~mm}$. With regard to the computational efficiency, it is important to point up that the accurate analysis of this multiport junction needed 0.9 seconds per frequency point.

In view of the results provided by the optimized design of the analyzed 
multiport waveguide junctions, it is possible to conclude a useful design guideline to estimate the maximum radius allowed for a certain electrical performance. The wide-band degradation of the designed devices is sensitive to the ratio $R / b$. On the one hand, we have observed that when the ratio $R / b$ is lower than 0.25 , the usable bandwidth of the device remains almost invariable and no further optimizations are required. On the other hand, when higher values of the ratio are considered, specially for $R / b>0.5$, an important degradation of the electrical response may be observed, and new optimized designs could be necessary. In this worst case $(R / b>0.5)$, we have concluded that it may be possible to recover a relative bandwidth under $-20 \mathrm{~dB}$ similar to the one obtained for the case in which $R=0$, as we have verified, for instance, in the case of $H$-plane bends. Even in the case of more complex structures (such as turnstile junctions), an optimized response can be designed obtaining an improved relative bandwidth.

Finally, the authors would like to point out that, after performing similar studies for $E$-plane bends and T-junctions (considering in these cases compensating posts and irises [3], 6]), similar conclusions to those reached for the corresponding $H$-plane cases have been obtained when mechanization effects are considered.

\section{Conclusion}

A new CAD tool for an accurate and efficient design of compensated multiport waveguide junctions considering the mechanization effects associated to low-cost production techniques has been proposed. It has been shown that, although the wide-band performance of the device can be reduced when high values of the radius of the rounded corners are considered, it is possible to compensate for this degradation by properly re-designing the compensating element, thus achieving an optimum design of these components. 


\section{Acknowledgements}

This work was supported by the Ministerio de Economía y Competitividad, Spanish Government, under the Research Project TEC2013-47037-C5-4-R.

Link to the article:

http://dx.doi.org/10.1016/j.aeue.2014.10.003

2014 Elsevier GmbH. All rights reserved.

\section{References}

[1] W. Wessel, T. Sieverding, F. Arndt, Mode-matching analysis of general waveguide multiportjunctions, in: IEEE MTT-S International Microwave Symposium Digest, 1999, pp. 1273-1276.

[2] S. Cogollos, V. E. Boria, P. Soto, A. A. San Blas, B. Gimeno, M. Guglielmi, Direct computation of the admittance parameters of a cubic junction with arbitrarily shaped access ports using the BI-RME method, IEE Proceedings on Microwaves, Antennas and Propagation 150 (2) (2003) 111-119.

[3] A. A. San Blas, F. Mira, V. E. Boria, B. Gimeno, M. Bressan, P. Arcioni, On the fast and rigorous analysis of compensated waveguide junctions using off-centered partial-height metallic posts, IEEE Transactions on Microwave, Theory and Techniques 55 (1) (2007) 168-175.

[4] A. Navarrini, R. L. Plambeck, A turnstile junction waveguide orthomode transducer, IEEE Transactions on Microwave, Theory and Techniques 54 (1) (2006) 272-277.

[5] Y. Aramaki, N. Yoneda, M. Miyazaki, T. Horie, Ultra-thin broadband OMT with turnstile junction, IEEE MTT-S International Microwave Symposium Digest (2003) 47-50.

[6] A. A. San Blas, F. Mira, V. E. Boria, B. Gimeno, M. Bressan, G. Conciauro, P. Arcioni, Efficient CAD of optimal multi-port junctions loaded with 
partial-height cylindrical posts using the 3D BI-RME method, in: IEEE MTT-S International Microwave Symposium Digest, 2005, pp. 67-70.

[7] S. Park, H. Lee, Y. Kim, A turnstile junction waveguide orthomode transducer for the simultaneus dual polarization radar, in: Asia-Pacific Microwave Conference, 2009, pp. 135-138.

[8] P. Arcioni, M. Bozzi, M. Bressan, G. Conciauro, L. Perregrini, Frequency/time-domain modeling of 3-D waveguide structures by a BIRME approach, International Journal of Numerical Modeling: Electronic Networks, Devices and Fields 15 (2002) 3-21.

[9] A. A. San Blas, F. J. Pérez, J. Gil, F. Mira, V. E. Boria, B. Gimeno, Full-wave analysis and design of broadband turnstile junctions, Progress in Electromagnetics Research Letters 24 (2011) 149-158.

[10] G. Gerini, M. Guglielmi, G. Lastoria, Efficient integral equation formulations for the computation of the multimode admittance or impedance matrix of planar waveguide junctions, in: IEEE MTT-S International Microwave Symposium Digest, 1998, pp. 1747-1750.

[11] J. Hirokawa, K. Sakurai, M. Ando, N. Goto, An analysis of a waveguide Tjunction with an inductive post, IEEE Transactions on Microwave, Theory and Techniques 39 (3) (1991) 563-566. 